\title{
Smartdust Network for Tactical Border Surveillance Using Multiple Signatures
}

\author{
Seema.C.Mohan ${ }^{1}$, S.Arulselvi ${ }^{2}$ \\ (Department of Electronics and Communication Engineering, Bharath University, Tamil Nadu, India).
}

\begin{abstract}
The paper aims at designing a system for border surveillance using the concept of smartdust technology. Smartdusts are small dust like wireless sensor motes with multiple on-board sensors and microcontroller. In real time, thousands of such smartdust motes must be deployed in a large area. The motes can form a network among them and has wireless connection to outside world. Onboard hardware include a variety of sensors for vibration/seismic, magnetic, acoustic and thermal signature recognition, a microcontroller for processing these sensor values and a radio transceiver for communication over a wireless network. In this experimental project, we have designed one central monitoring mote and two smartdust motes using ARM controller, IEEE radio transceiver and sensors.The central monitoring mote also displays the tracking history of intrusion on enabling a switch.
\end{abstract}

Keywords:Advanced RISC Machine (ARM), Cortex microcontroller software interface standard (CMSIS), Inter Integrated Circuit (I $\left.{ }^{2} C\right)$, MEMS accelerometer, Serial Peripheral Interface (SPI),Smartdust mote.

\section{Introduction}

The greatest threat to national security is "Terrorism", infiltrating through borders. In critical border areas, regular forces or even satellites cannot monitor the intruding terrorists, as the area monitored is quite large and complex. This paper proposes an innovative and effective solution to this problem by designing a next generation, intelligent, ultra-small, dust like, wireless sensor motes with multiple onboard sensors and a processor for detecting enemy intrusion across borders and battlefields. As the project is conceptualized on 'smartdust technology', a brief elucidation of smartdust definition is provided.

Smartdusts are dust size devices which are light in weight [1]. Each smartdust mote can be considered as a tiny computer with one or more sensors, on-board power supply, a communication system and a controller. It can communicate with other smartdust devices using the wireless radio network. The battery life of a smartdust mote can vary from a few hours to ten years depending on the size and capability of the device. A common mote communication scheme uses radio frequency signals to communicate over relatively short distances. This allows designers to minimize mote size and reduce power consumption. When communicating, the devices pass each message to a neighbouring mote, which, in turn, passes the message onto a neighbouring mote, and so on, until the message reaches the destination ie the central monitoring mote.The network of motes continue to perform even if some of its communicaiton paths fail to operate. And once a mote is placed in an existing network, it adapts to blend in with the other nodes to form a larger network; and when a mote fails, the other devices in the network take over its load.Fig. 1 shows smartdusts deployed in a forest.

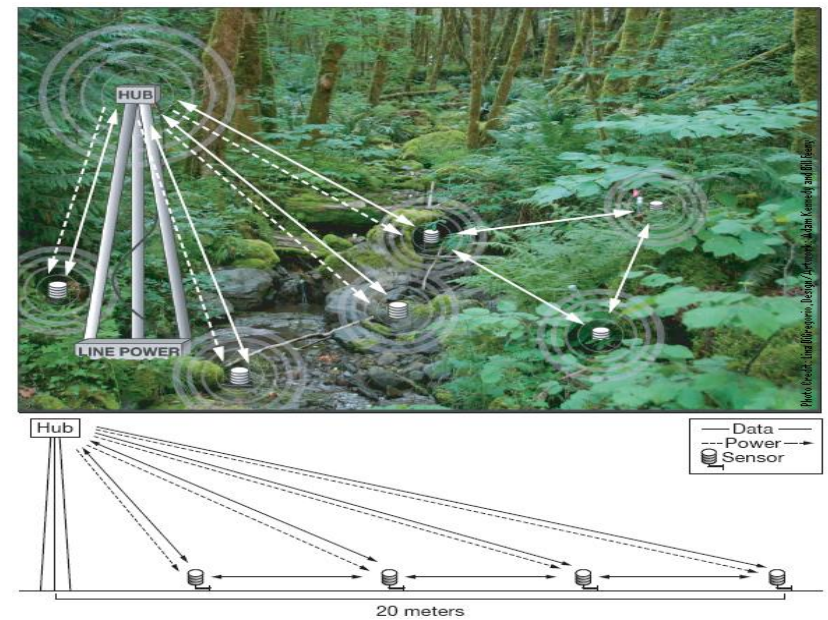

Figure 1: Smartdust deployed in a forest 
In real time, thousands of such motes are deployed in the field for detecting intrusion but in the project we are employing only two smartdust sensor motes and a single monitoring mote on an experimental basis.

There are several existing border surveillance systems or methods in use today [2]. The most popular methods are flying aircraft, deploying armed forces and ground surveillance using RADARS. The drawbacks of these systems are discussed here;(a)Flying aircraft: the aircraft is usually run only for limited hours or for one or two days. (b) Deploying armed forces: it is very difficult for the armed forces to monitor a large area with great precision. Also, it is very unsafe and risky for the forces to remain at difficult terrains such as dense forests, snow covered or mountain areas for days where bad weather conditions prevail. (c) Ground surveillance using RADAR:No single RADAR can fulfill all border surveillance requirements with single RADAR equipment. There is search RADAR that does the search function and once the search is done we would need the track RADAR for the tracking. There is also complex RADAR equipment that could integrate two or more RADAR functions in one RADAR instrument.RADAR as a whole is very huge and bulky. RADAR's are very much visible owing to its big size and hence, it is subject to enemy detection.Due to the RADAR's bulkiness we would require one or two men to carry it to the place of deployment or it is mounted on an armed vehicle. The smartdust concept may be implemented in border surveillance applications in an effective and efficient way to solve many of these problems as we shall see in the coming sections.

\subsection{Related Work on border surveillance}

There are several works carried out in the area of border surveillance. As an example, the study conducted by T.J.Nohara explains the use of commercial approach to the deployment of radar surveillance[3]. The literature says, "surveillance solutions must be multi-mission suitable, scalable, flexible, maintainable, upgradeable, interoperable, shareable, and affordable", which is very true when it comes to border surveillance and other security systems. Smartdust system satisfies the above mentioned features and its compact size is an added advantage when deployed in the battlefields. To give another example, the work done by C.Neumann and his colleagues explains about the protection of our borderlines as well as military camps using Radar surveillance methods[4]. The challenges of remote border monitoring have been detailed in the work conducted by P.Pratap and his colleagues[5]. The paper discusses three major issues to be addressed to build an effective ground surveillance system and the issues are 'providing reliable and efficient power', 'providing adequate and timely maintenance to minimize downtime' and 'networking systems for effective data transmission'. Concluding, the work says that a system that overcomes these challenges will provide a "cost-effective solution requiring minimal support infrastructure solution to meet border monitoring and protection needs." Smartdust system ensures that it meets these challenges, to be discussed in the following sections. Numerous other works were carried out in designing border surveillance systems and also improving on the existing methodologies. Most of the works carried out was about improving on the existing Radar technologies, using unmanned air vehicles and fiber optic sensing [3][4][6][7].This paper proposes a system based on smartdusts for border surveillance applications that can help solve many of the challenges posed by conventional systems especially concerned to power consumption, maintainability, safety and coverage.

The system structure of the smartdust networks is presented in the next section followed by the hardware and software design in the third and fourth sections. The third section also details about the components used and it's set up. The software design section also includes a high level flow chart for the system and the forthcoming section describe the output obtained when the system was put under test. The final section describes the features, few concerns and some enhancements of the proposed project.

\section{System Structure}

The project aims to develop a system of two motes that communicates with a central monitoring mote. The system structure can be broadly classified into two- smatdust mote circuit and the central monitoring mote circuit. The block diagram for the smartdust network is shown in Fig. 2. The system consists of the following components/modules:

a) Microcontroller: 32-bit ARM Cortex-M3 microcontroller, to control the smartdust mote.

b) Display: Graphics LCD, to show the intrusion type and tracking history.

c) Communication: IEEE 802.15.4 wireless protocol, for communication between motes.

d) Thermal sensor: Enhanced PIR sensor, to sense the acoustic sound signals.

e) Vibration sensor: 3-axis MEMS accelerometer, to sense the acoustic sound signals.

f) Acoustic sensor: MEMS Microphone is used to sense the acoustic sound signals.

g) Magnetic sensor: Intruders carrying weapons and moving in vehicles can be identified using their magnetic signature in this AMR Magnetic sensor. 

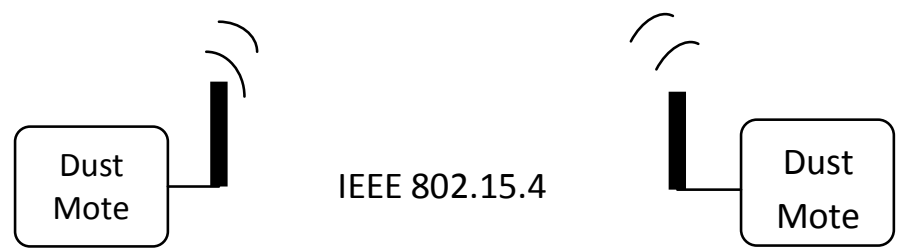

Network

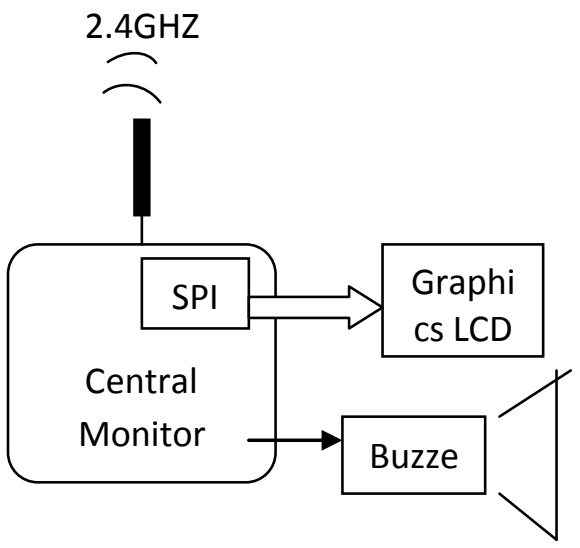

Figure 2: Block diagram of smartdust network

Each smartdust mote consists of a variety of sensors like magnetic, thermal, acoustic and vibration sensors for detecting the respective signatures. The controller present in the dust mote processes these values and sends them over the wireless network using IEEE802.15.4 protocol. The structure designed for a smartdust mote is shown in Fig. 3.

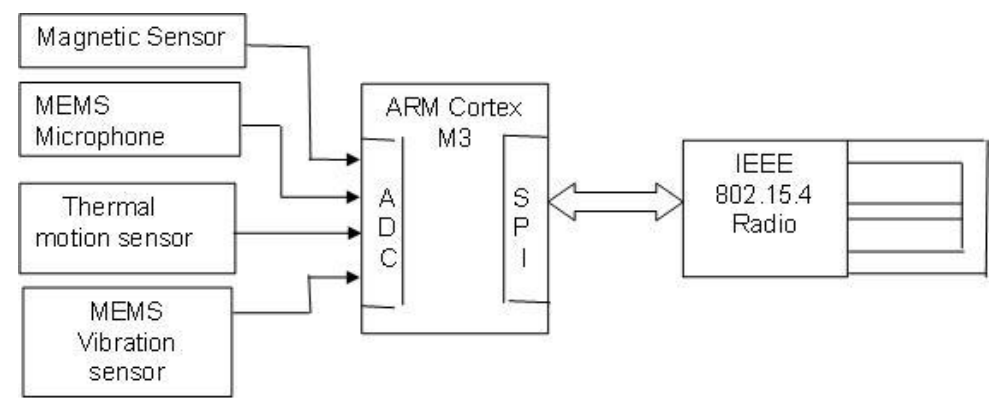

Figure 3:Proposed block diagram ofsmartdust mote

\section{Hardware Design}

The major hardware used in the project are ARM controller, sensors, transceiver, graphics display, buzzer, crystal and power supply. The project intends to develop two smartdust mote boards and one central monitoring mote. A list of the components used for the project work can be found in Table 1.

Table 1: List of components

\begin{tabular}{lll}
\hline Component & Specification & Number \\
\hline ARM controller & ARM LPC1313 & 3 \\
Vibration sensor & PHIDGETS 1104 & 2 \\
Magnetic sensor & KMA199E & 2 \\
MEMS microphone & SPM0404HE5H & 2 \\
PIR sensor & SB0081 & 2 \\
IEEE802.15.4 radio transceiver & MRF24J40 & 3 \\
Graphics display & NOKIA 5110 & 1 \\
Crystal & 12 MHz & 3 \\
Buzzer & - & 1 \\
\hline
\end{tabular}


Other componenets used are LEDs and switches as per need.

The circuit diagram for the central monitoring mote and smartdust mote are shown in Fig. 4 and Fig.5 respectively.

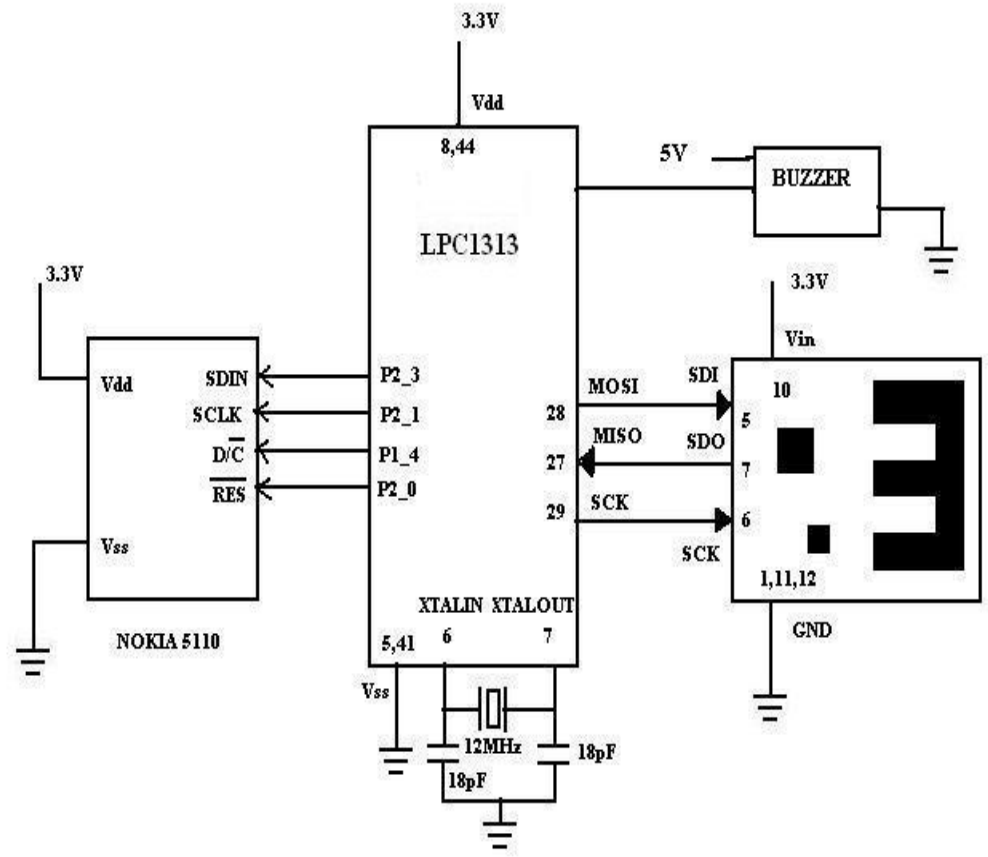

Figure 4: Circuit diagram of central monitoring mote

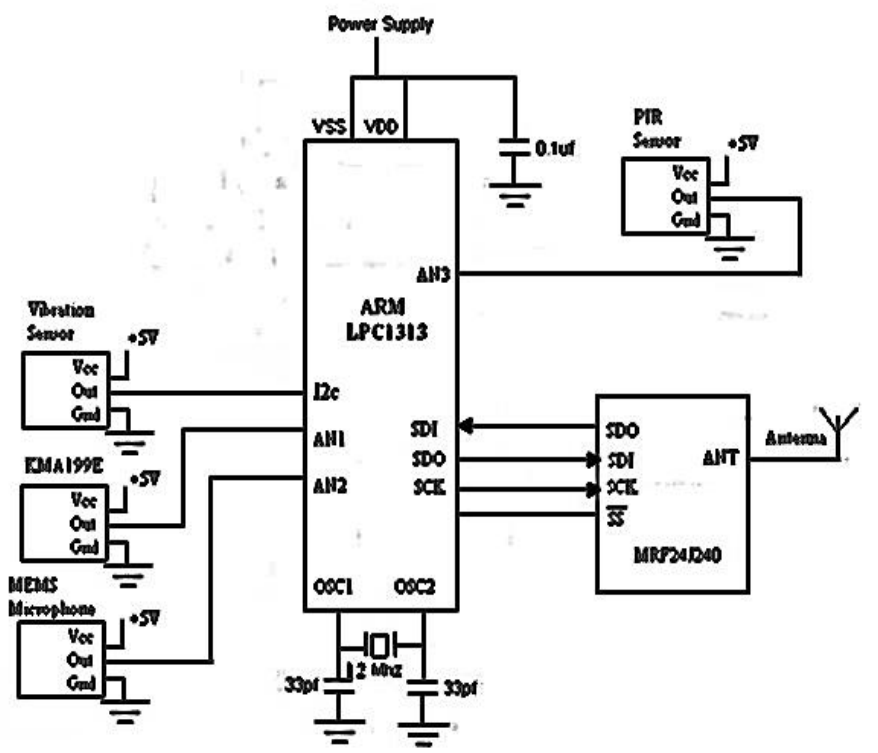

Figure 5: Circuit diagram of smartdust mote

Each of the key components used in this work are detailed here.

i. ARM Controller - The project uses ARM Cortex M3 controller, which is the next generation 32 bit ARM processor for embedded applications based on ARMv7-M architecture. The specialty of this ARM controller is its Harvard architecture [8][9]. The separate instruction and data buses allow parallel instruction fetching and data storage.The ARM Cortex - M3 controller has been chosen in this work for the following reasons: better energy efficiency, more functionality out of battery life and ability to meet 
increasing energy demands for low energy products when compared to other controllers [8].It also has the smallest code size for any microcontroller. Reducing the code size is the key to squeezing your application code into minimum amount of flash [8].

ii. Magnetic sensor - The magnetic sensor used is KMA199E which is a magnetic angle sensor system (numbered 3 in Fig.6). The MagnetoResistive (MR) sensor bridges and the mixed signal Integrated Circuit (IC) are integrated into a single package. This angular measurement system KMA199E is preprogrammed, pre-calibrated and therefore, ready to use. The KMA199E allows user specific adjustments of angular range, zero angle and clamping voltages [10]. The settings are stored permanently in an Electrically Erasable Programmable Read-Only Memory (EEPROM).

iii. MEMS Microphone - MEMS microphone is similar to the standard ECMs (Electro condenser microphones) found in modern consumer electronics, except that the components are built onto a single chip using CMOS technology, rather than assembled from discrete parts. It can sense the acoustic signals [11]. The MEMS microphone used is SPM0404HE5H(numbered 2 in Fig.6).

iv. PIR Motion sensor - The motion detector module used is SB0081, which is a pyroelectric sensor module developed for human body detection (numbered 1 in Fig.6). A PIR detector combined with a Fresnel lens are mounted on a compact size PCB together with an analog IC, SB0081, and limited components to form the module [12]. It has a very compact size and operates at $3.3 \mathrm{~V}$.

v. Vibration sensor - It is a piezoelectric transducer, which when displaced from the mechanical neutral axis; the bending creates strain within the piezoelectric element and generates voltage (numbered 4 in Fig.6). We have used PHIDGETS 1104 type in the project. The type of measurement used by the device is ratiometric [13].

vi. Transceiver - The MRF24J40 is an IEEE 802.15.4 ${ }^{\mathrm{TM}}$ Standard compliant $2.4 \mathrm{GHz}$ RF transceiver [14]. The MRF24J40 creates a low-cost, low-power, low data rate (250 or $625 \mathrm{kbps})$ Wireless Personal Area Network (WPAN) device (numbered 5 in Fig.6). The MRF24J40 interfaces many popular microcontrollers via a 4-wire serial SPI interface, interrupt, wake and Reset pins. The MRF24J40 provides hardware support for energy detection, Carrier Sense, CSMA-CA Algorithm, Automatic Packet Retransmission, Automatic Acknowledgment. The Wireless Personal Area Network (WPAN) focuses on low-cost, low-speed ubiquitous communication between devices. The range of operation is 10-100 meter and provides a transfer rate of $250 \mathrm{Kbit} / \mathrm{s}$. It uses CSMA/CA for collision avoidance. It supports secure communications and employs peer -to-peer or star topology. The operating frequency band is $2400-2483.5 \mathrm{MHz}$ with up to sixteen channels. It uses Offset Quadrature Phase Shift Keying (OQPSK) modulation.

vii. Graphics LCD -The Graphics LCD used is Nokia 5110, which is a basic LCD screen for lots of applications. It is mounted on an easy to solder PCB [15]. It uses the PCD8544 controller which is a low power CMOS LCD controller/driver designed to drive a graphics display of 48 rows and 84 columns. This is interfaced to the ARM microcontroller through serial bus interface. The logic supply voltage range VDD to VSS is 2.7 to $3.3 \mathrm{~V}$.

The system has been assembled in three boards, one each for the two sensor motes and one for the monitoring mote. The experimental set up of the proposed system is shown in Fig. 6 and Fig.7.

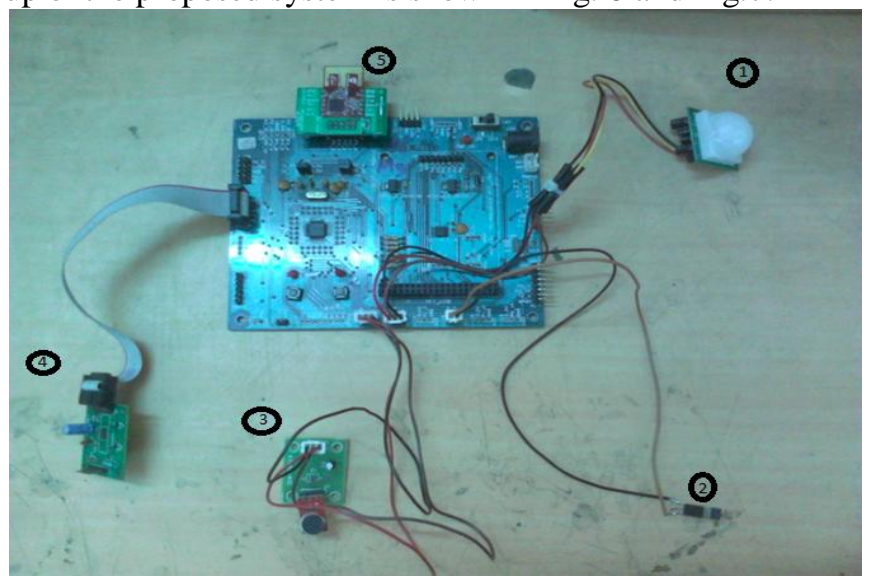

Figure 6: Smartdust mote with sensors 


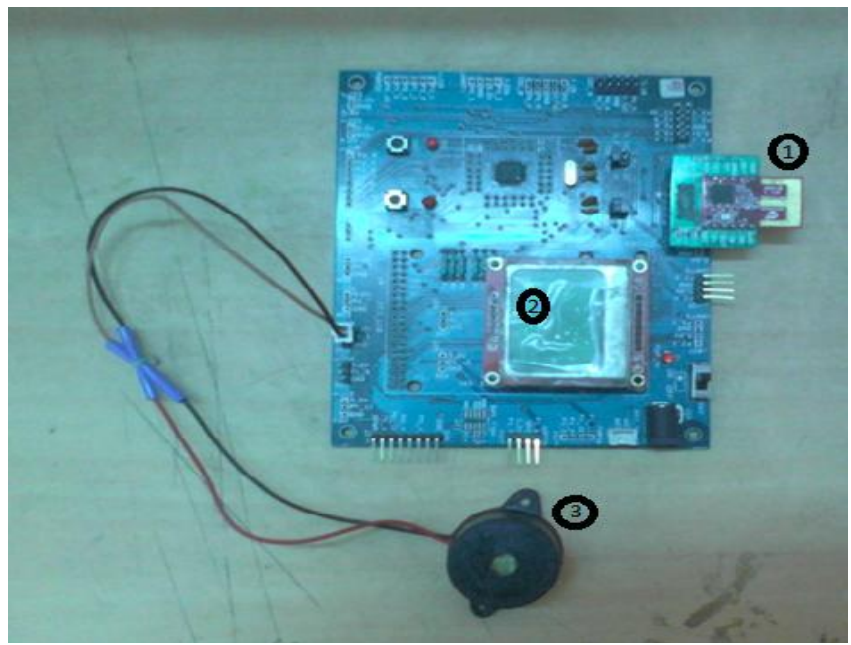

Figure 7:Smartdust monitoring mote

The MEMS microphone is connected to port AD0, metal sensor to AD2, PIR sensor to AD3, MEMS accelerometer (vibration sensor) to $\mathrm{I}^{2} \mathrm{C}$ port and transceiver through SPI.

\section{Software Design}

This section details about the software aspects of the project. The development environment used is LPCXpresso IDE, which is a suitable development tool for the LPC1000 series of ARM Cortex-M microcontrollers.It is a complete tool chain for LPC1000 series of Cortex-M microcontrollers. The different software's used in the project work are CMSIS (Cortex microcontroller software interface standard) from ARM, IEEE 802.15.4 protocol stack, $\mathrm{I}^{2} \mathrm{C}$ and SPI protocol drivers. The application code programming is done in embedded C.CMSIS is a vendor independent hardware abstraction layer for the Cortex-M processor series. It enables consistent and simple software interfaces to the processor and the peripherals thus; simplifying software reuse.Creation of software is a major cost factor in the embedded industry. Standardizing the software interfaces across all Cortex-M silicon vendor products, would lead to significant cost reduction when creating new projects or migrating existing software to a new device. IEEE 802.15.4 protocol stackis the standard that specifies the physical layer and media access control for low-rate wireless personal area networks. It provides low cost, low speed communication between devices. Here, the emphasis is on low cost communication of nearby devices with little or no underlying infrastructure. $\mathrm{I}^{2} \mathrm{C}$ protocol driveris a two- wire interface standard. It is used to attach low-speed peripherals to the controller. It uses Serial Data Line (SDA), Serial Clock (SCL). The bus speed is $100 \mathrm{Kbit} / \mathrm{s}$.SPI protocol driveris a serial peripheral interface and is widely used with embedded systems because it is simple and efficient interface. It has three signal wires that hold a clock (SCK), a "Master Out Slave In" (MOSI) data line, and a "Master In Slave Out". A high level process flow chart for the project is given in Fig.8 and Fig.9. 


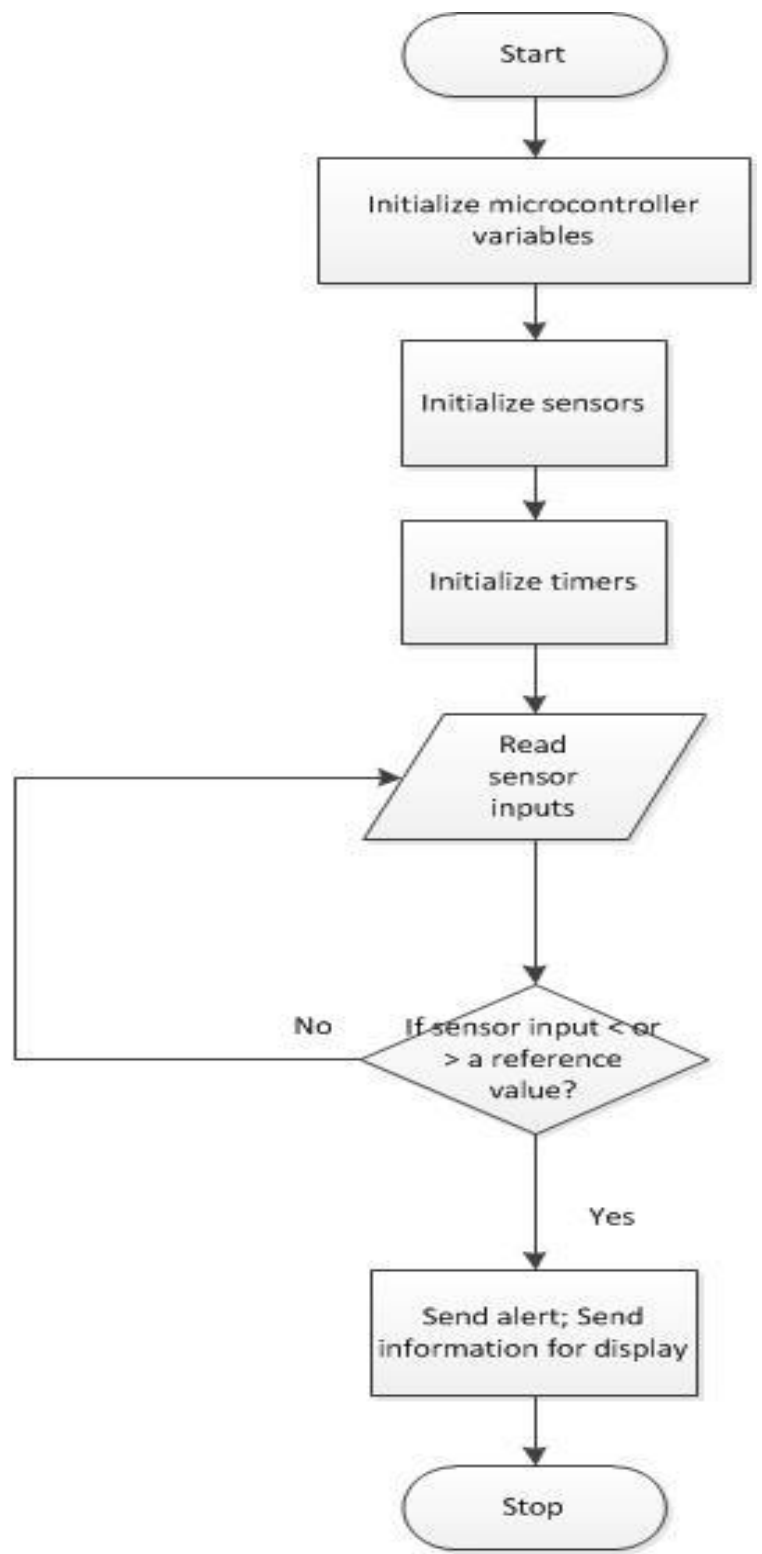

Figure 8:Flow chart for sensor motes 


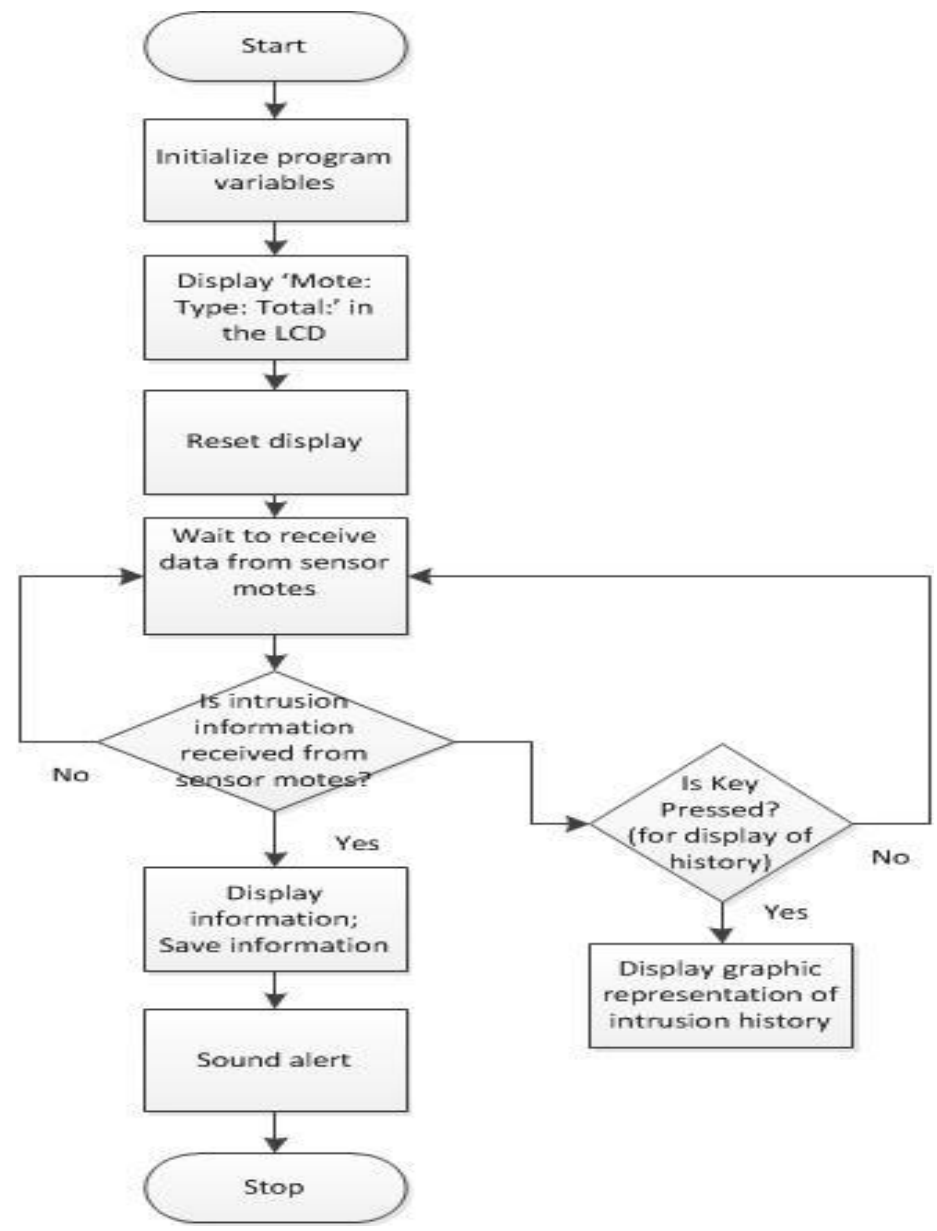

Figure 9: Flow chart for the monitoring mote

\section{Result}

The sensor motes were placed at a distance of 10 meters (test distance) apart and the monitoring mote placed in its vicinity. On powering each of these boards using the on-board battery, the test LED in the boards were switched ON indicating that wireless connection has been established between them. On giving the inputs, (i)Presence of human, (ii) Human motion, (iii) Human speech and (iv) Movement of a metallic bar, near the sensors, it was found to be detected by the sensors and communicated over to the monitoring mote which displayed which mote detected what (type of input) in the graphics display. It also displayed the total number of inputs it has sensed just to give an idea about the concentration or the strength of intrusion in that area. The output obtained is shown in Fig.10 and Fig.11. The display in Fig.10 shows that the detection of intrusion has happened near mote ' 1 ' and the type of input has been 'human motion'. It also shows a total of 6 which indicates that it is the sixth time an intrusion were detected in its vicinity. Similarly, Fig.11 shows that mote 1 has detected presence of metal near its vicinity and it was the ninth time it has detected an intrusion.

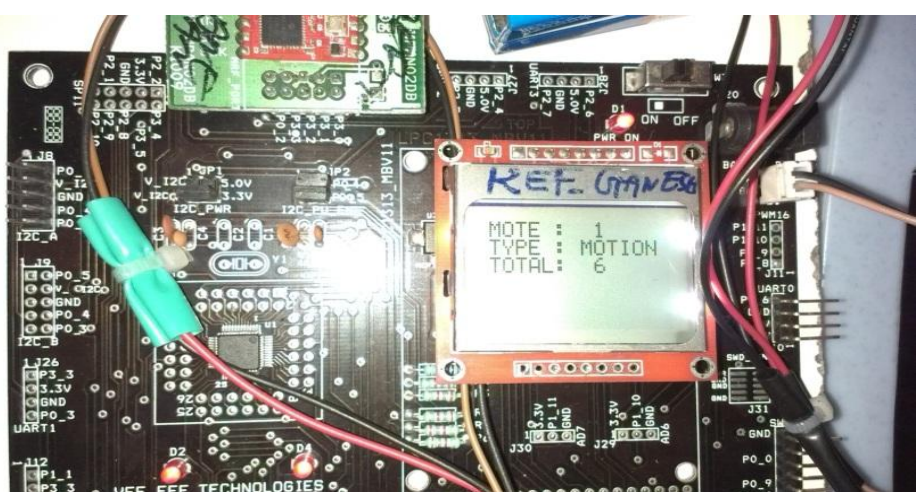

Figure 10: Monitoring mote displaying intrusion, Sample 1. 


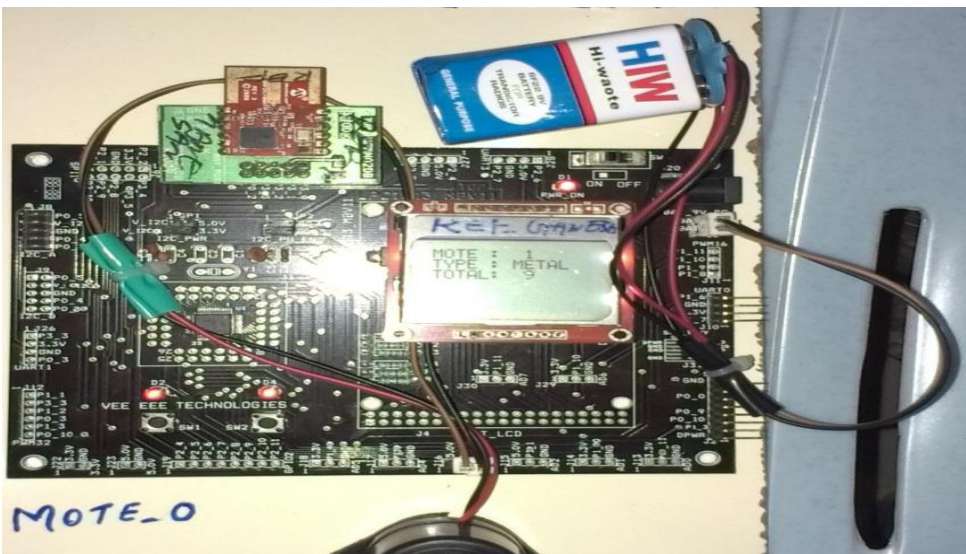

Figure 11: Monitoring mote displaying intrusion, Sample 2.

The system also displays a graph, Fig.12 that indicates the intrusion history by showing which mote has displayed the intrusion on the press of a switch. It approximately gives an idea as to where or near which mote the intrusion is maximum.

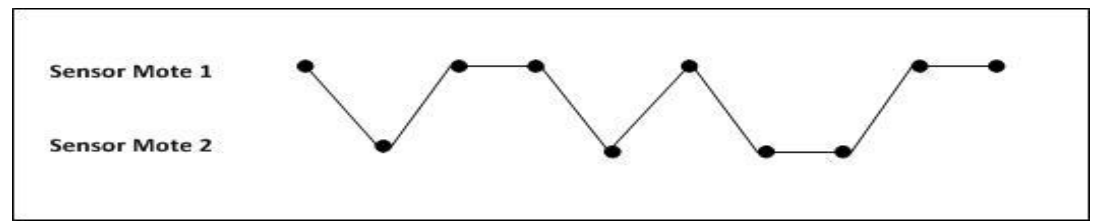

Figure 12: Graph showing intrusion history

\section{System features}

Relying on the concept of using smartdust networks in border surveillance, thousands of motes may be deployed in any terrain conditions, even inside a dense forest where satellite monitoring is impossible. The smartdusts does not require any maintenance as once deployed they can run for many years. They are not easily prone to enemy detection compared to their conventional counterparts. Variety of onboard sensors makes the mote to identify any kind of intrusion, humans, machines or vehicles. The intrusion path can be tracked using wireless communication between motes. Low power 32-bit ARM Cortex-M3 microcontroller enables the mote to be operated for years from battery power. A portable monitoring node with graphics LCD is used for easy UI where the intrusion path will be plotted graphically and alarm is raised.

\section{Conclusion}

The proposed system of smartdusts for border surveillance applications was designed, developed and tested in the laboratory. Few suggestions for the betterment of the project would be to make a distinction between animals or humans that is intruding based on the comparison of temperatures. PIR sensor can make out the difference in the body temperatures or the heat from animal and human bodies. Another major point of apprehension would be the availability of the smartdust chips.Smartdust motes are not yet available on a large scale and even if they do most of the motes are of the size of a deck of cards. We could hope for the future motes to be of dust size at the same time available at a reasonable rate. The key downsides of using smartdust networks in border surveillance is the pollution it causes because once deployed the smartdust mote remain in the soil for years. Therefore, let us hope for greener smartdust mote circuits to be developed on a large scale. Also, solar powered batteries can be of great benefit to the smartdust circuits as the circuits once deployed must remain in soil for years without maintenance. Another area of enhancement that we could suggest is that the smartdust motes could be made to give details such as the position of the intrusion and the weather conditions at the place of intrusion.

\section{References}

[1] Doug Steel, Smartdust, UH ISRC Technology briefing, March 2005.

[2] Pratap.P, Kallberg J.M, Thomas L.A,Challenges of remote border monitoring, 2010 IEEE International Conference on Technologies for Homeland Security (HST)

[3] Nohara, T.J;A commercial approach to successful persistent radar surveillance of sea, air and land along the northern Border, 2010 IEEE International Conference on Technologies for Homeland Security (HST)

[4] Neumann, C.; Weiss, G.; Wahlen, A.; Brehm, T:Ground Surveillance With Mmw Radar For Border Control And Camp Protection Applications, EuropeanMicrowave Conference, 2007. 
[5] Pratap, P.; Kallberg, J.M.; Thomas, L.A;Challenges Of Remote Border Monitoring, 2010 IEEE International Conference on Technologies for Homeland Security (HST)

[6] Girard, A.R.; Howell, A.S.; Hedrick, J.K;Border Patrol And Surveillance Missions Using Multiple Unmanned Air Vehicles, 2004. CDC. 43rd IEEE Conference on Decision and Control

[7] Owen, Arch; Duckworth, Gregory; Worsley, Jerry, Optasense: Fibre Optic Distributed Acoustic Sensing For Border Monitoring, 2012 EuropeanIntelligence and Security Informatics Conference (EISIC)

[8] ShyamSadasivan, An introduction to the ARM Cortex-M3 Processor, October 2006, Open journal

[9] Cortex-M3 Technical reference manual from ARM, 2010, http://www.arm.com

[10] NXP Semiconductors, KMA199E, Programmable sensor, Rev.01-18, October 2007, http://www.nxp.com

[11] Knowles Acoustics, SPM0404HESH-PB specification, 2009

[12] SB0081, Product manual

[13] Phidgets, 1104_0_product_manual_September 30, 2009

[14] IEEE, MRF24J40, http://www.microchip.com

[15] PCD85441,ProductSpecification,1999, http://www.semiconductors.phillips.com 